\title{
Role of enhancer activation in pancreatic cancer metastasis
}

\author{
Paloma Cejas ${ }^{1,2,3}$, Marta Mendiola ${ }^{3,4,5}$, Jaime Feliu ${ }^{2,3,6,7}$ \\ ${ }^{1}$ Center for Functional Cancer Epigenetics, Dana-Farber Cancer Institute, Boston, Massachusetts, USA; ${ }^{2}$ Translational Oncology Lab, IdiPAZ, La \\ Paz University Hospital, Madrid, Spain; ${ }^{3}$ CIBERONC CB16/12/00398, La Paz University Hospital, Madrid, Spain; ${ }^{4}$ Molecular Pathology Section, \\ Institute of Medical and Molecular Genetics (INGEMM) La Paz University Hospital, Madrid, Spain; ${ }^{5}$ Molecular Pathology and Therapeutic Targets \\ Lab, IdiPAZ, La Paz University Hospital, Madrid, Spain; ${ }^{6}$ Cátedra UAM-AMGEN, Autonomous University of Madrid, Madrid, Spain; ${ }^{7}$ Clinical \\ Oncology Department, La Paz University Hospital, Madrid, Spain \\ Correspondence to: Paloma Cejas; Jaime Feliu. Institute for Health Research (IdiPAZ), Paseo de la Castellana 261, 28046, Madrid, Spain. \\ Email: paloma.cejas@idipaz.es; jaime.feliu@salud.madrid.org. \\ Comment on: Roe JS, Hwang CI, Somerville TDD, et al. Enhancer Reprogramming Promotes Pancreatic Cancer Metastasis. Cell 2017;170:875-888.e20.
}

Submitted Mar 14, 2018. Accepted for publication Mar 27, 2018.

doi: $10.21037 /$ tcr.2018.04.02

View this article at: http://dx.doi.org/10.21037/tcr.2018.04.02

Aberrancies in chromatin conformation can lead to altered gene expression thus promoting cancer development. Chromatin is the substratum for transcription factors (TF) to bind DNA and the degree of chromatin compaction determines TF accessibility to their target cis-regulatory elements including promoters and enhancers. Results comparing chromatin profiles between primary tumors and matched non-malignant tissues evidence a profound dynamic throughout the carcinogenic process (1-4). This dynamic effectively reprograms the enhancer landscape toward the activation of oncogenes such as $M Y C(1,2)$. Supporting findings in a variety of tumors of different lineages suggest that the aberrant enhancer reprogramming is a general mechanism involved in carcinogenesis (1-4). However, the role that this mechanism could have in the metastatic process remains vastly unexplored.

Pancreatic ductal adenocarcinoma (PDAC) is one of the most lethal human malignancies due to absence of methods for early diagnosis and chemoresistance of advanced disease. KRAS is mutated in $95 \%$ of PDACs and is a well-validated driver of PDAC initiation and growth $(5,6)$. However, the biology involved in the advance to metastasis is less understood. In a recent article, Roe et al. (7) tested the hypothesis that enhancer reprogramming could be a mechanism promoting PDAC progression. To that aim, the authors performed a genomewide analysis of histone $\mathrm{H} 3$ lysine 27 acetylation (H3K27ac) in cultured organoids from a well-established mouse model of PDAC progression (8). The analysis included organoids derived from pancreatic ducts ( $\mathrm{N}$ organoids), Pancreatic Intraepithelial lesions (P organoids) and pairs of Primary tumor ( $\mathrm{T}$ ) and Metastasis ( $\mathrm{M}$ organoids) providing a complete picture of the enhancer landscape across the disease.
The results show a significant divergence in the enhancer landscape only observed for the $\mathrm{M}$ organoids as compared to all the earlier stages that show highly correlated H3K27ac profiles (7). The lack of an evident enhancer reprogramming already for $\mathrm{T}$ organoids is somehow unexpected in view of previous $\mathrm{H} 3 \mathrm{~K} 27 \mathrm{ac}$ results in primary tumors (1-4,9). For colorectal and gastric cancer, also sharing with PDAC the gastrointestinal origin, H3K27ac landscape shows significant differences already in the primary tumor as compared to matched normal tissue, with a high number of newly activated GAIN enhancers nearby oncogenes $(1,2)$. The delayed enhancer reprogramming observed in PDAC derived organoids could be a tumortype specific characteristic or could result from differences between the mouse model and the primary human tissues. Mouse models are induced systems that share features of human cancer, although they represent a simplified version of a more complex disease. Therefore, direct analysis of human PDAC will be required to assess the relevancy of enhancer reprogramming in real patients.

To further investigate the involvement of this epigenetic mechanism in metastasis promotion, Roe et al. (7) focused on the GAIN enhancers in the transition between the $\mathrm{T}$ and the $M$ organoids that may be hypothesized as being directly involved in metastasis promotion. Interestingly, those $\mathrm{H} 3 \mathrm{~K} 27$ ac GAIN enhancers in M organoids already show accessible chromatin in the T organoids, by ATACseq analysis. This result suggests that enhancers may be in a predetermined but "poised" stage already in the primary tumor that could be switched on later to promote the metastatic process. The GAIN enhancers are close to genes for developmental pathways and show enrichment in 
binding motifs for Forkhead families (FOX) TFs. FOXA1 is overexpressed in $\mathrm{M}$ organoids and ChIP-seq analysis confirmed elevated occupancy of FOXA1 at the GAIN enhancers. FOXA1 is a pioneer TF that could potentially activate the H3K27ac GAIN enhancers during transition from $\mathrm{T}$ to $\mathrm{M}$ organoids. To assess that hypothesis, Roe et al. performed FOXA1 perturbation of expression in 2-dimensional (2D) organoid cultures. FOXA1 overexpression in $\mathrm{T}$ organoids resulted in the activation of a subset of the H3K27ac GAIN regions observed in $M$ organoids. This effect was enhanced by combined overexpression of FOXA1 and GATA5 in T organoids which coordinately resulted in a better recapitulation of the GAIN enhancers in $\mathrm{M}$ organoids. The activation of the GAIN enhancers was also accompanied by an increase in expression of the corresponding genes revealing the functionality of enhancer reprogramming. FOXA1 expression also resulted in an acquisition of a significant metastatic phenotype as shown by an enhanced anchorageindependent growth and enhanced ability of the 2D culture to colonize the lung parenchyma when injected via tail vein into recipient mice.

All together, the results from Roe et al. (7) highlight the crucial role that chromatin remodeling has on the PDAC metastatic process, implicating FOXA1 as the driver of enhancer reprogramming. Oncogenic alterations in the enhancer landscape could confer a fitness advantage to tumor cells leading to an increased metastatic behavior as a result of transcriptional mechanisms compatible with dedifferentiation and increased proliferation. The findings could have clinical implications for cancer therapy since new drugs that act on chromatin remodelers are currently in clinical trials and may benefit PDAC patients.

\section{Acknowledgments}

Funding: This work was supported by Instituto de Salud Carlos III the Spanish Economy and Competitiveness Ministry (grant PI13-01818).

\section{Footnote}

Provenance and Peer Review: This article was commissioned and reviewed by the Section Editor Xiaotian Sun (Department of Internal Medicine, Clinic of August First Film Studio, Beijing, China).

Conflicts of Interest: All authors have completed the ICMJE uniform disclosure form (available at http://dx.doi. org/10.21037/tcr.2018.04.02). The authors have no conflicts of interest to declare.
Ethical Statement: The authors are accountable for all aspects of the work in ensuring that questions related to the accuracy or integrity of any part of the work are appropriately investigated and resolved.

Open Access Statement: This is an Open Access article distributed in accordance with the Creative Commons Attribution-NonCommercial-NoDerivs 4.0 International License (CC BY-NC-ND 4.0), which permits the noncommercial replication and distribution of the article with the strict proviso that no changes or edits are made and the original work is properly cited (including links to both the formal publication through the relevant DOI and the license). See: https://creativecommons.org/licenses/by-nc-nd/4.0/.

\section{References}

1. Cohen AJ, Saiakhova A, Corradin O, et al. Hotspots of aberrant enhancer activity punctuate the colorectal cancer epigenome. Nat Commun 2017;8:14400.

2. Ooi WF, Xing $\mathrm{M}, \mathrm{Xu} \mathrm{C}$, et al. Epigenomic profiling of primary gastric adenocarcinoma reveals super-enhancer heterogeneity. Nat Commun 2016;7:12983.

3. Yao X, Tan J, Lim KJ, et al. VHL Deficiency Drives Enhancer Activation of Oncogenes in Clear Cell Renal Cell Carcinoma. Cancer Discov 2017;7:1284-305.

4. Cejas P, Li L, O'Neill NK, et al. Chromatin immunoprecipitation from fixed clinical tissues reveals tumor-specific enhancer profiles. Nat Med 2016;22:685-91.

5. Ying H, Kimmelman AC, Lyssiotis CA, et al. Oncogenic Kras maintains pancreatic tumors through regulation of anabolic glucose metabolism. Cell 2012;149:656-70.

6. Collins MA, Bednar F, Zhang Y, et al. Oncogenic Kras is required for both the initiation and maintenance of pancreatic cancer in mice. J Clin Invest 2012;122:639-53.

7. Roe JS, Hwang CI, Somerville TDD, et al. Enhancer Reprogramming Promotes Pancreatic Cancer Metastasis. Cell 2017;170:875-888.e20.

8. Boj SF, Hwang CI, Baker LA, et al. Organoid models of human and mouse ductal pancreatic cancer. Cell 2015;160:324-38.

9. Cejas P, Cavazza A, Yandava CN, et al. Transcriptional Regulator CNOT3 Defines an Aggressive Colorectal Cancer Subtype. Cancer Res 2017;77:766-79.

Cite this article as: Cejas P, Mendiola M, Feliu J. Role of enhancer activation in pancreatic cancer metastasis. Transl Cancer Res 2018;7(Suppl 4):S519-S520. doi: 10.21037/ tcr.2018.04.02 